\title{
Associations between cerebrospinal fluid biomarkers and long-term neurologic outcome in dogs with acute intervertebral disk herniation
}

\author{
Tige H. Witsberger, DVM, DACvs; Jonathan M. Levine, DVM, DACVIM; \\ Geoffrey T. Fosgate, DVM, PhD, DACVPM; Margaret R. Slater, DVM, PhD; Sharon C. Kerwin, DVM, MS, DACvs; \\ Karen E. Russell, DVM, PhD, DACVP; Gwendolyn J. Levine, DVM, DACVP
}

\begin{abstract}
Objective-To examine associations between CSF biomarkers, initial neurologic dysfunction, and long-term ambulatory outcome in dogs with acute intervertebral disk herniation (IVDH).

Design-Prospective clinical study.

Animals - 54 dogs with acute thoracolumbar IVDH and 16 clinically normal dogs.

Procedures-For each dog, variables, including CSF myelin basic protein (MBP), lactate, calcium, glucose, and total protein concentrations; nucleated cell count; and creatine kinase (CK) and aspartate aminotransferase activities, were measured. For dogs with thoracolumbar IVDH, initial neurologic function was characterized by use of a modified Frankel score (MFS; determined on a scale of 0 to 5 , where 0 represented paraplegia with no deep nociception and 5 represented paraspinal hyperesthesia only). Long-term follow-up was assessed.
\end{abstract}

Results-Among dogs with thoracolumbar IVDH, those with CSF CK activity $\leq 38 \mathrm{U} / \mathrm{L}$ had a 35-fold increase in the odds of long-term ambulation, compared with the odds in dogs with CSF CK activity > $38 \mathrm{U} / \mathrm{L}$, adjusting for neurologic functioning at the evaluation. The CSF lactate, calcium, and glucose concentrations and aspartate aminotransferase activity were not predictive of long-term ambulatory outcome. Data analysis revealed that longterm functional recovery was $>98 \%$ for affected dogs, regardless of their initial MFS, when CSF CK activity was $\leq 38 \mathrm{U} / \mathrm{L}$ and MBP concentration was $\leq 3 \mathrm{ng} / \mathrm{mL}$.

Conclusions and Clinical Relevance-In dogs with acute thoracolumbar IVDH, CSF CK activity and MBP concentration appeared to be prognostic indicators and, along with initial MFS, can be used to predict long-term ambulatory outcome. (J Am Vet Med Assoc 2012;240:555-562)

$\mathrm{T}$ raditionally, preoperative pelvic limb nociceptive status has been used to determine long-term functional outcome in dogs undergoing surgery to treat thoracolumbar IVDH. ${ }^{1-10}$ Affected dogs with intact pelvic limb nociception have reported rates of postsurgical voluntary ambulation that range from $86 \%$ to $96 \%$, whereas $43 \%$ to $76 \%$ of dogs lacking pelvic limb deep nociception are able to walk voluntarily following surgery. ${ }^{1-10}$ Although nociceptive evaluation will always remain a simple and easily performed assessment, it is imperfect at determining the risk of therapeutic failure, cannot distinguish structural versus functional lesions, and does not provide biological data that could inform targeted treatment strategies. ${ }^{3,6-10}$

Magnetic resonance imaging findings and results of assessments of certain CSF biomarkers offer objective, independent means of determining postsurgical out-

\begin{tabular}{ll}
\hline & \multicolumn{1}{c}{ AbBREVIATIONS } \\
AST & Aspartate aminotransferase \\
CI & Confidence interval \\
CK & Creatine kinase \\
IVDH & Intervertebral disk herniation \\
MBP & Myelin basic protein \\
MFS & Modified Frankel score \\
MR & Magnetic resonance \\
T2W & T2-weighted \\
\hline
\end{tabular}

come in dogs with thoracolumbar IVDH. For example, the presence of hyperintensity within the spinal cord parenchyma in T2W MR images has been shown to predict long-term ambulatory outcome in dogs with thoracolumbar IVDH. ${ }^{11,12}$ In 1 study, ${ }^{12}$ the measured length of hyperintensity in the spinal cord observed in sagittal

\footnotetext{
From the Departments of Veterinary Small Animal Clinical Sciences (Witsberger, JM Levine, Kerwin), Veterinary Integrative Biosciences (Slater), and Veterinary Pathobiology (Russell, GJ Levine), College of Veterinary Medicine and Biomedical Sciences, Texas A\&M University, College Station, TX 77843; and the Department of Production Animal Studies, Faculty of Veterinary Science, University of Pretoria, Onderstepoort 0110 , South Africa (Fosgate). Dr. Slater's present address is American Society for the Prevention of Cruelty to Animals, 1717 S Philo Rd, Ste 36, Urbana, IL 61802.

Supported by American Kennel Club ACORN Grant No. 1180-A.

Presented as a poster at the American College of Veterinary Surgeons Symposium, Washington, DC, October 2009.

The authors thank Julie Harris, Alisha Onkst, and Amanda Garner for assistance with data collection.

Address correspondence to Dr. Witsberger (twitsberger@cvm.tamu.edu).
} 
T2W MR images obtained from dogs with IVDH was divided by the length of the L2 vertebra to generate a standardized ratio (the T2W length ratio). Findings of that study revealed that each unit increase in the T2W length ratio was associated with a nearly 2 -fold reduction in the odds of recovery, independent of neurologic assessment. More recently, dogs with acute thoracolumbar IVDH that had CSF concentrations of MBP $\geq$ $3 \mathrm{ng} / \mathrm{mL}$ were reported ${ }^{13}$ to have 0.09 times the odds of ambulation at a follow-up examination, compared with affected dogs that had CSF concentrations of MBP less than this cutoff value. Other investigators ${ }^{14}$ have shown that CSF pleocytosis and abnormally high CSF protein concentration are associated with severe physical examination-based features of spinal cord injury in dogs with thoracolumbar IVDH.

Lactate, calcium, CK, and AST have been detected in CSF samples collected from animals with various CNS diseases. In cats with experimental spinal cord injury, CSF lactate and calcium concentrations were high, compared with those in control animals; associations with prognosis were not determined. ${ }^{15}$ In 1 study ${ }^{16} \mathrm{CSF}$ CK activity in dogs with thoracolumbar IVDH was increased, compared with that in unaffected control dogs, but attempts were not made to correlate activity with functional outcome. Other researchers have not found CSF CK activity to be a useful determinant of disease status or outcome in humans ${ }^{17}$ or horses. ${ }^{18}$ In humans with head trauma, ${ }^{19}$ CSF AST activity has been associated with lesion severity, and in dogs, ${ }^{20}$ it has been detected in individuals with degenerative diseases of the CNS.

Cerebrospinal fluid lactate, calcium, and glucose concentrations and CK and AST activities can be evaluated in a point-of-care manner in most veterinary clinics. The purpose of the prospective study reported here was to determine whether CSF lactate, calcium, and glucose concentrations and CK and AST activities were associated with the presence of acute thoracolumbar IVDH in dogs and whether those variables could be used to predict the long-term functional outcome of those affected dogs. We hypothesized that higher CSF concentrations or activities of these biomarkers would be negatively associated with ambulatory outcome. In addition, we examined the correlation of the concentrations and activities of selected biomarkers in CSF samples with those in plasma samples obtained from dogs with acute thoracolumbar IVDH.

\section{Materials and Methods}

Dogs with acute thoracolumbar IVDH-Between March and December 2008, nonambulatory dogs admitted to Texas A\&M University Veterinary Teaching Hospital with an acute history of presumed thoracolumbar IVDH were prospectively recruited for participation in the study and another investigation..$^{13}$ Owner consent was obtained prior to dog enrollment by use of standard documents approved by the Clinical Research Review Committee. To be included in the affected group, dogs had to meet certain criteria as follows: signs of nonambulatory paraparesis or paraplegia with a $\leq 7$-day history of neurologic impairment, intervertebral disk-associated spinal cord compression located between vertebral articulations at T3 through L5, and confirmation of disk herniation during surgery or necropsy. Exclusion criteria were as follows: no diagnostic imaging evidence of thoracolumbar IVDH, excessive blood contamination of CSF samples (> 13,200 RBCs/ $\mu \mathrm{L}^{21}$ ), and assessment of CSF biomarkers $>1$ hour after sample acquisition. It was expected that 60 affected dogs would be recruited during the study period and assumed that $85 \%$ of dogs would have a successful long-term outcome; this provided a statistical test with $80 \%$ power to detect a risk ratio of 3.5 (ie, dogs with certain biomarker concentrations or activities being 3.5 times as likely to have an unsuccessful outcome as dogs without those biomarker concentrations or activities) as significant at the 5\% level.

Dogs without acute thoracolumbar IVDH (controls)-Sixteen research colony dogs that did not have acute thoracolumbar IVDH were used as control dogs. Sample collection from these dogs was approved by the Institutional Animal Care and Use Committee of Texas A\&M University. For inclusion of a dog in the control group, results of a physical examination, neurologic evaluation, $\mathrm{CBC}$, and plasma biochemical analysis had to be within reference intervals.

Procedures for dogs with acute thoracolumbar IVDH-For each of the affected dogs, age, sex, breed, duration of clinical signs prior to hospital admission, prereferral administration of glucocorticoids (yes or no), prereferral administration of NSAIDs (yes or no), and days of hospitalization following surgery were recorded. Dogs were classified as chondrodystrophoid or nonchondrodystrophoid on the basis of previously established criteria. ${ }^{22}$ Complete physical and neurologic examinations were performed on all affected dogs on arrival at the hospital. A blood sample ( $2 \mathrm{~mL}$ ) was routinely collected into a tube containing lithium heparin. Neurologic dysfunction was classified according to the MFS as follows: paraspinal hyperesthesia only (grade 5), ambulatory paraparesis and ataxia (grade 4), nonambulatory paraparesis (grade 3), paraplegia with intact nociception (grade 2), paraplegia with no superficial nociception (grade 1), and paraplegia with no deep nociception (grade 0).22,23

After premedication of each affected dog, anesthesia was induced via IV administration of $\operatorname{propofol}^{a}$ and anesthesia was maintained by use of inhalation anesthetic agents (sevoflurane ${ }^{\mathrm{b}}$ or isoflurane ${ }^{\mathrm{c}}$ ) for diagnostic imaging and collection of a CSF sample from the cerebromedullary cistern. Advanced imaging comprised myelography, computed tomography, or MR imaging; the type of imaging performed depended on access to equipment and clinician preference. When T2W spinal cord hyperintensity was present, the $\mathrm{T} 2 \mathrm{~W}$ length ratio (measured on a single view that contained the most hyperintensity observed in sagittal T2W MR images) was measured. ${ }^{12}$ One sample of CSF $(1 \mathrm{~mL})$ was collected (within 18 hours after hospital admission) from each dog by a surgery resident (THW), board-certified surgeon (SCK), or board-certified neurologist (JML).

Thoracolumbar IVDH was confirmed at surgery (hemilaminectomy or pediculectomy) or necropsy. Routine postoperative care (including administration of opioid analgesics, urinary bladder management, pelvic limb passive range of motion exercises, and physical re- 
habilitation) was performed for all dogs as needed until discharge from the hospital. Owners were instructed to confine their dogs for 4 to 6 weeks after surgery and continue postoperative care (passive range of motion exercises and rehabilitation) as needed.

Procedures for dogs without acute thoracolumbar IVDH-For each of the control dogs, age, sex, and breed were recorded. Dogs were classified as chondrodystrophoid or nonchondrodystrophoid on the basis of previously established criteria. ${ }^{22}$ During the neurologic examination performed to ensure that dogs met the criteria for inclusion in the control group, dogs were classified according to the MFS. For study analyses, a blood sample ( 3 to $5 \mathrm{~mL}$ ) was collected into a tube containing lithium heparin from each dog.

Each control dog was anesthetized for collection of a CSF sample (1 mL) from the cerebromedullary cistern, as described for the affected dogs. Samples were collected by a surgery resident (THW).

Plasma analyses-Plasma obtained from the blood samples from dogs with thoracolumbar IVDH and control dogs was analyzed within 1 hour after collection. Plasma lactate, glucose, and calcium concentrations and AST and CK activities were determined in each sample by use of an automated chemistry analyzer. ${ }^{\mathrm{d}}$

CSF analyses-Cerebrospinal fluid samples obtained from dogs with thoracolumbar IVDH and control dogs were used for various study analyses. Approximately 1 to $1.5 \mathrm{~mL}$ of CSF from each dog was placed in a plasma tube and submitted for cytologic examination, assessments of cell counts, and total protein concentration determination. In addition, 2 to 4 additional aliquots ( 200 to $300 \mu \mathrm{L}$ ) of CSF from each dog were frozen at $-80^{\circ} \mathrm{C}$ immediately after collection for determination of MBP concentration as previously described. ${ }^{13}$ Samples were analyzed within 1 hour after collection by use of an automated chemistry analyzer. ${ }^{\mathrm{d}}$ Variables of interest included nucleated cell count; lactate, calcium, glucose, and total protein concentrations; and CK and AST activities. Cerebrospinal pleocytosis was defined as a CSF nucleated cell count $\geq 5$ cells $/ \mu \mathrm{L}$. Type of pleocytosis was defined by predominance of a particular cell type (> 60\% of cells present) or when $>40 \%$ of cells present were of 1 cell type and no other cell type composed $>33 \%$ of the cells present. ${ }^{24}$ The reference interval for CSF total protein concentration was $<27 \mathrm{mg} / \mathrm{dL}$.

An adjunct experiment was performed to determine whether frozen samples could be used as surrogates for fresh CSF samples. Samples of CSF were frozen at $-80^{\circ} \mathrm{C}$ and thawed at room temperature (approx $22^{\circ} \mathrm{C}$ ) before analysis. Duration of freezing ranged from 17 to 78 days. Cerebrospinal fluid lactate, glucose, and calcium concentrations and AST and CK activities were determined in each sample by use of an automated chemistry analyzer. ${ }^{d}$ The values for those 5 CSF variables derived from frozen samples were compared with values derived from the fresh CSF samples.

Long-term follow-up of dogs with acute thoracolumbar IVDH-All dogs that were alive were assessed for voluntary ambulation $>3$ months after discharge from the hospital. Ambulatory status was determined via in-hospital examination or through information derived from a questionnaire sent via e-mail or mailed to owners. Nonresponders were contacted by telephone. Final outcome was classified as successful when a dog regained the ability to walk voluntarily at any point during the study without a recorded relapse. Final outcome was classified as unsuccessful when a dog did not recover ambulation during a minimum 3-month follow-up period or did not recover ambulation and was euthanized.

Statistical analysis-Descriptive statistics including medians and ranges were calculated for ordinal and continuous variables such as biomarker concentrations or activities, age, and MFS at the time of the initial evaluation and, for dogs with thoracolumbar IVDH, duration of clinical signs at the time of the initial evaluation and the $\mathrm{T} 2 \mathrm{~W}$ length ratio of spinal cord hyperintensity on T2W MR images. Categorical variables, including chondrodystrophic breed, sex, and CSF characteristics and, for dogs with thoracolumbar IVDH, preoperative treatments, were summarized by counts, percentages, and mid-P exact 95\% CIs. Continuous variables were compared across groups (control dogs, dogs with successful outcomes, and dogs with unsuccessful outcomes) by use of Kruskal-Wallis tests overall with significant differences followed by pairwise Mann-Whitney $U$ tests with Bonferroni adjustment for multiple comparisons. Categorical variables were compared across groups by use of Pearson $\chi^{2}$ tests with significant differences followed by pairwise $\chi^{2}$ or Fisher exact tests with Bonferroni adjustment of $P$ values.

The correlation of CSF biomarker concentrations with MFS at admission to the hospital, duration of clinical signs at the time of the initial evaluation, or T2W length ratio of spinal cord hyperintensity was assessed on the basis of calculated values of Spearman $\rho$. The correlations between plasma and each of the CSF biomarker concentrations or activities were also estimated by use of Spearman $\rho$ values. Receiver-operating characteristic curve analysis was performed to determine optimal CSF biomarker concentrations or activities that could be used to predict a successful long-term outcome in dogs with confirmed thoracolumbar IVDH. The cutoff that maximized the Youden index (ie, sensitivity + specificity -1 ) was selected as optimal.

Bivariable logistic regression was used to determine whether CSF biomarker concentrations or activities significantly predicted long-term functional outcome. Cerebrospinal fluid biomarkers were dichotomized on the basis of the optimal cutoff identified from the receiver-operating characteristic analysis, and biomarkers for which the Wald $P$ value was $<0.20$ were considered possible predictors and retained for further analysis. Multivariable logistic regression was used to estimate the ability of CSF biomarker concentrations or activities to predict a long-term successful outcome, adjusting for disease severity and other potential predictors. Quantitative variables that violated the assumption of linearity in the natural logarithm of the odds were modeled as categorical variables. The assigned MFS at the time of admission to the hospital was forced into all models to adjust for disease severity. A backward stepwise ap- 
proach (based on Wald tests) was used to evaluate the ability of variables to predict a successful outcome, and Hosmer and Lemeshow tests were used to assess the fit of multivariable models. Predictive probabilities for the determination of prognosis were calculated from parameter estimates of the final multivariable model.

All analyses were performed by use of commercially available software ${ }^{e}$ except for the tabular analyses. ${ }^{f}$ Results were interpreted at the 5\% level of significance unless stated otherwise.

\section{Results}

Descriptive findings-A total of 62 nonambulatory client-owned dogs were initially enrolled in the affected group. Five of the dogs were excluded for various reasons: 1 because of neoplasia, 1 because of suspected myelomalacia detected via diagnostic imaging with no surgery or necropsy performed, 2 because of excessive blood contamination of the collected CSF samples $(14,850$, and $292,788 \mathrm{RBCs} / \mu \mathrm{L})$, and 1 because the CSF sample was not analyzed within the first hour after collection (analyzed at day 10). Long-term follow-up could not be obtained in 3 dogs that lacked voluntary ambulation at the time of discharge from the hospital (2 had an MFS of 3, and 1 had an MFS of 0 ). Consequently, 54 dogs with acute thoracolumbar IVDH were included in the final outcome analysis.

Most of the affected dogs were Standard and Miniature Dachshunds $(\mathrm{n}=35)$. The remaining breeds included mixed $(n=4)$, Maltese (2), Pembroke Welsh Corgi (2), and 1 each of 11 other breeds. Median age of these dogs was 5 years (range, 1.5 to 13 years). The group included 7 sexually intact males, 6 sexually intact females, 21 castrated males, and 20 spayed females. At the initial evaluation, all dogs were nonambulatory. Median initial MFS among the 54 affected dogs was 2 (range, 0 to 3); initial MFS was 3 in 24 dogs, 2 in 17 dogs, 1 in 4 dogs, and 0 in 9 dogs. Median duration of neurologic signs prior to initial evaluation was 1 day (range, 1 to 7 days). Prior to referral, glucocorticoids had been administered to 16 dogs and NSAIDs had been administered to 10 dogs.
Diagnostic imaging included myelography for 18 dogs, computed tomography for 10 dogs, and MR imaging for 26 dogs. Disk-associated compressive lesions were detected at 8 articulations; the T12-13 ( $n=14)$, T13-L1 (10), and T11-12 (10) articulations were most commonly affected. With regard to surgical procedures, 2 dogs underwent pediculectomy and 52 dogs underwent hemilaminectomy. Median duration of hospitalization was 8 days (range, 2 to 45 days). Fifty dogs were alive at time of discharge from the hospital; in this group, MFS was 0 in 4 dogs, 3 in 22 dogs, and 4 in 24 dogs. At long-term follow-up, 45 of 54 dogs were voluntarily ambulatory. There were 9 dogs with unsuccessful outcomes; 5 of these dogs were euthanized prior to follow-up. Among those 5 dogs, ascendingdescending myelomalacia was confirmed at necropsy in 3 . On the basis of surgery type, successful outcomes were achieved in 2 of 2 dogs that underwent pediculectomy and 43 of 52 dogs that underwent hemilaminectomy. The proportion of dogs with successful outcomes was not different between surgery types (pediculectomy vs hemilaminectomy).

Among the 16 control dogs, there were 7 Labrador Retrievers, 5 mixed-breed dogs, 2 Red Bone Hounds, and 2 Blue Tick Hounds. The median age of the dogs was 3 years (range, 2 to 4 years). The control group included 13 castrated males and 3 spayed females. Control dogs had a significantly $(P<0.05)$ lower median age than did dogs with thoracolumbar IVDH that had successful outcomes (Table 1). Control dogs were also more likely to be nonchondrodystrophoid, compared with all dogs with thoracolumbar IVDH (Table 2). No control dogs had CSF pleocytosis or high CSF total protein concentration.

Plasma biomarkers-Plasma CK activity was greater than the reference limit in a significantly higher proportion of dogs with thoracolumbar IVDH, compared with control dogs (Table 2). Inadvertent errors in sample submission resulted in a small number of the dogs for which plasma CK (11 dogs) and AST (7 dogs) activities and lactate ( 1 dog) concentration were not determined. The proportion of dogs for which values of

Table 1-Median age, initial MFS, duration of clinical signs, and CSF characteristics for 54 dogs with thoracolumbar IVDH (confirmed at surgery) and 16 control dogs without thoracolumbar IVDH at a single referral hospital.

\begin{tabular}{|c|c|c|c|c|c|c|}
\hline \multirow[b]{3}{*}{ Variable } & & & \multicolumn{4}{|c|}{ Dogs with thoracolumbar IVDH } \\
\hline & \multicolumn{2}{|c|}{ Control dogs $(n=16)$} & \multicolumn{2}{|c|}{ Successful outcome (n= 45) } & \multicolumn{2}{|c|}{ Unsuccessful outcome $(n=9)$} \\
\hline & Median & Range & Median & Range & Median & Range \\
\hline Age $(y)^{*}$ & $3^{\text {a }}$ & $2-4$ & $5^{b}$ & $2-12$ & $6^{a, b}$ & $1.5-13$ \\
\hline Initial MFS* & $6^{\mathrm{a}}$ & NA & $2^{\mathrm{b}}$ & $0-3$ & $0^{c}$ & $0-3$ \\
\hline Duration of signs (d) & NA & NA & 1 & $1-7$ & 2 & $1-3$ \\
\hline \multicolumn{7}{|l|}{ CSF characteristic } \\
\hline Nucleated cell count (cells/uL)* & $0^{\mathrm{a}}$ & $0-2$ & $2^{\mathrm{b}}$ & $0-35$ & $7^{\mathrm{b}}$ & $1-245$ \\
\hline Total protein concentration (mg/dL) & 27 & $22-35$ & 25 & $12-56$ & 36 & $13-110$ \\
\hline CK activity (U/L)* & $<20^{\mathrm{a}}$ & $<20-42$ & $20^{\mathrm{a}}$ & $<20-174$ & $62^{\mathrm{b}}$ & $<20-115$ \\
\hline AST activity (U/L) & 18 & $15-24$ & 19 & $12-37$ & 22 & $16-29$ \\
\hline Lactate concentration $(\mathrm{mg} / \mathrm{dL})$ & 12.0 & $10.2-14.2$ & 11.5 & $7.3-17.5$ & 12.7 & $8.8-17.9$ \\
\hline Calcium concentration (mg/dL) & 5.0 & $4.9-11.4$ & 5.0 & $4.5-5.8$ & 5.1 & $4.8-5.5$ \\
\hline Glucose concentration (mg/dL)* & $58.5^{\mathrm{a}}$ & $56-64$ & $73^{\mathrm{b}}$ & $55-108$ & $76^{b}$ & $70-108$ \\
\hline
\end{tabular}


plasma lactate concentration and AST activity exceeded the upper reference limit was not different between dogs with and without thoracolumbar IVDH. Similarly, the proportion of dogs for which values of plasma glucose and calcium concentrations exceeded the upper reference limit was not different between dogs with and without thoracolumbar IVDH (data not shown). In dogs with thoracolumbar IVDH, concentrations of lactate $(\rho=0.35 ; P=0.003)$, calcium $(\rho=0.36 ; P=0.002)$, and glucose $(\rho=0.69 ; P<0.001)$ in plasma and CSF were significantly correlated, although the strength of correlation varied from poor to good (Table 3 ). There was no significant relationship between plasma and CSF CK or AST activities.

CSF characteristics and relationships between those findings and outcome for dogs with thoracolumbar IVDH-In the pilot experiment to evaluate selected variables in fresh versus frozen CSF samples from 10 dogs with acute IVDH, there was no correlation between CK activity in fresh and frozen CSF samples (data not reported). As a result, only data from fresh CSF samples were used in the study.

In CSF samples obtained from the 54 dogs with thoracolumbar IVDH, the median nucleated cell count was 3 cells/ $\mu \mathrm{L}$ (range, 0 to 245 cells $/ \mu \mathrm{L}$ ), median RBC count was 21 cells $/ \mu \mathrm{L}$ (range, 0 to 10,780 cells $/ \mu \mathrm{L}$ ), and median total protein concentration was $26 \mathrm{mg} / \mathrm{dL}$ (range, 12 to $110 \mathrm{mg} / \mathrm{dL}$ ). Cerebrospinal fluid pleocyto- sis was evident in 16 dogs and classified as neutrophilic in 11 dogs, mixed cell in 4 dogs, and large mononuclear in $1 \mathrm{dog}$. Cerebrospinal fluid total protein concentration was high ( $\geq 27 \mathrm{mg} / \mathrm{dL})$ in 25 dogs. Control dogs had a significantly $(P<0.05)$ lower median CSF nucleated cell count $(0$ cells/ $\mu \mathrm{L})$ than did affected dogs with either successful ( 2 cells/ $\mu \mathrm{L})$ or unsuccessful $(7$ cells $/ \mu \mathrm{L})$ outcomes. However, median CSF nucleated cell count did not significantly differ between dogs with successful and unsuccessful outcomes (Table 1).

The median CSF CK activity did not differ between control dogs and dogs with thoracolumbar IVDD that had a successful outcome. However, median CSF CK activity in dogs with thoracolumbar IVDH that had an unsuccessful outcome was significantly increased (62 $\mathrm{U} / \mathrm{L})$, compared with values in control dogs $(<20 \mathrm{U} / \mathrm{L})$ and dogs with thoracolumbar IVDH that had a successful outcome (20 U/L). The median CSF glucose activity was higher in dogs with thoracolumbar IVDH, compared with control dogs. Median CSF lactate and calcium concentrations and AST activity were not related to the presence or absence of thoracolumbar IVDH.

Receiver-operating characteristic curve analysis suggested an optimal CSF CK activity cutoff value of $38 \mathrm{U} / \mathrm{L}$. By use of this value and a previously reported ${ }^{13}$ cutoff value for CSF MBP concentration of $3 \mathrm{ng} / \mathrm{mL}$ in the same group of dogs, both CSF CK activity and MBP concentration were significant predictors of long-term outcome in dogs with thoracolumbar IVDH (Table 4).

Table 2-Proportions and 95\% Cls for various characteristics among 54 dogs with thoracolumbar IVDH (confirmed at surgery) and 16 control dogs without thoracolumbar IVDH at a single referral hospital.

\begin{tabular}{|c|c|c|c|c|c|c|}
\hline \multirow[b]{3}{*}{ Variable or characteristic } & & & \multicolumn{4}{|c|}{ Dogs with thoracolumbar IVDH } \\
\hline & \multicolumn{2}{|c|}{ Control dogs $(n=16)$} & \multicolumn{2}{|c|}{ Successful outcome ( $n=45)$} & \multicolumn{2}{|c|}{ Unsuccessful outcome $(n=9)$} \\
\hline & Proportion & $95 \% \mathrm{CI}$ & Proportion* & $95 \% \mathrm{Cl}$ & Proportion* & $95 \% \mathrm{Cl}$ \\
\hline Female & 0.19 & $0.05-0.43$ & 0.44 & $0.30-0.59$ & 0.67 & $0.33-0.91$ \\
\hline Neutered & 1.0 & $0.83-1.0$ & 0.73 & $0.59-0.85$ & 0.89 & $0.56-0.99$ \\
\hline Chondrodystrophic breed $\dagger$ & $0.0^{\mathrm{a}}$ & $0.0-0.17$ & $0.93^{\mathrm{b}}$ & $0.83-0.98$ & $0.89^{\mathrm{b}}$ & $0.56-0.99$ \\
\hline CSF pleocytosis & $0.0^{\mathrm{a}}$ & $0.0-0.17$ & $0.22^{\mathrm{b}}$ & $0.12-0.36$ & $0.67^{\mathrm{a}}$ & $0.33-0.91$ \\
\hline High plasma CK activity $†$ & $0.0^{\mathrm{a}}$ & $0.0-0.17$ & $0.49^{\mathrm{b}}(37)$ & $0.33-0.65$ & $0.50^{\mathrm{b}}(6)$ & $0.15-0.85$ \\
\hline High plasma AST activity & 0.44 & $0.22-0.68$ & $0.73(40)$ & $0.57-0.85$ & $0.86(7)$ & $0.47-0.99$ \\
\hline High plasma lactate concentration & 0.0 & $0.0-0.17$ & 0.11 (44) & $0.04-0.23$ & 0.11 & $0.01-0.44$ \\
\hline Glucocorticoid administration & NA & NA & 0.33 & $0.21-0.48$ & 0.11 & $0.01-0.44$ \\
\hline NSAID administration & NA & NA & 0.16 & $0.07-0.28$ & 0.33 & $0.09-0.67$ \\
\hline
\end{tabular}

Table 3-Biomarker concentrations and activities in plasma and CSF samples collected from 54 dogs with thoracolumbar IVDH (confirmed at surgery) and 16 control dogs without thoracolumbar IVDH at a single referral hospital.

\begin{tabular}{|c|c|c|c|c|c|c|}
\hline \multirow[b]{2}{*}{ Biomarker } & \multicolumn{2}{|l|}{ Plasma } & \multicolumn{2}{|l|}{ CSF } & \multicolumn{2}{|c|}{ Correlation } \\
\hline & $\begin{array}{c}\text { Median } \\
\text { (No. of samples) }\end{array}$ & Range & $\begin{array}{c}\text { Median } \\
\text { (No. of samples) }\end{array}$ & Range & Spearman $\rho$ & $P$ value \\
\hline $\begin{array}{l}\text { CK activity }(\mathrm{U} / \mathrm{L}) \\
\text { AST activity }(\mathrm{U} / \mathrm{L}) \\
\text { Lactate concentration }(\mathrm{mg} / \mathrm{dL}) \\
\text { Calcium concentration }(\mathrm{mg} / \mathrm{dL}) \\
\text { Glucose concentration }(\mathrm{mg} / \mathrm{dL})\end{array}$ & $\begin{array}{r}189(59) \\
42(63) \\
14.4(70) \\
9.9(70) \\
104(70)\end{array}$ & $\begin{array}{l}43-4,438 \\
18-216 \\
1.6-68.4 \\
4.4-11.9 \\
62-177\end{array}$ & $\begin{array}{r}20(70) \\
19(70) \\
11.9(70) \\
5.0(70) \\
72(70)\end{array}$ & $\begin{array}{c}0-174 \\
12-37 \\
7.3-17.9 \\
4.5-11.4 \\
55-108\end{array}$ & $\begin{array}{l}0.108 \\
0.029 \\
0.330 \\
0.378 \\
0.702\end{array}$ & $\begin{array}{r}0.417 \\
0.822 \\
0.005 \\
0.001 \\
<0.001\end{array}$ \\
\hline
\end{tabular}


Table 4-Results of multivariable logistic regression analyses for the prediction of successful clinical outcome for 54 dogs with thoracolumbar IVDH (confirmed at surgery).

\begin{tabular}{|c|c|c|c|}
\hline Variable & Parameter estimate $(\beta)$ & $P$ value (Wald) & Odds ratio $(95 \% \mathrm{CI})$ \\
\hline CSF CK activity $\leq 38 \mathrm{U} / \mathrm{L}$ & 3.55 & 0.024 & $35(1.6-757)$ \\
\hline CSF MBP concentration $\leq 3 \mathrm{ng} / \mathrm{mL}$ & 3.49 & 0.016 & $33(1.9-554)$ \\
\hline Initial MFS & - & 0.096 & - \\
\hline 0 or 1 & Referent & - & - \\
\hline 2 & 3.54 & 0.042 & $35(1.1-1,055)$ \\
\hline 3 & 2.02 & 0.124 & $7.5(0.6-98)$ \\
\hline
\end{tabular}

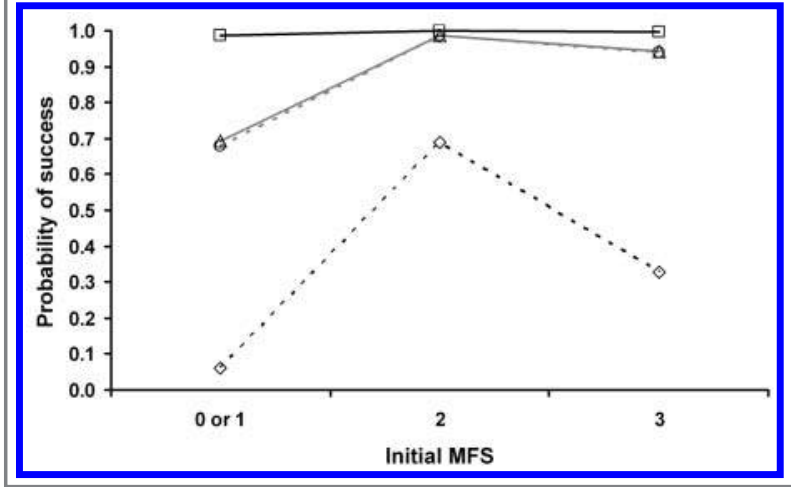

Figure 1-Estimated probability of a successful clinical outcome based on results of multivariable logistic regression analysis of the MFS and CSF concentrations of CK and MBP in 54 dogs with thoracolumbar IVDH (confirmed during surgery) at a single referral hospital. Dogs were grouped on the basis of CSF CK activity and MBP concentration as follows ( 3 dogs had missing CSF MBP information): $\geq 38 \mu \mathrm{g} / \mathrm{mL}$ and $\geq 3 \mathrm{ng} / \mathrm{mL}$, respectively ( $=9$; diamonds); $\geq 38 \mu \mathrm{g} /$ $\mathrm{mL}$ and $<3 \mathrm{ng} / \mathrm{mL}$, respectively (14; circles); $<38 \mu \mathrm{g} / \mathrm{mL}$ and $\geq 3 \mathrm{ng} /$ $\mathrm{mL}$, respectively (9; triangles); or $<38 \mu \mathrm{g} / \mathrm{mL}$ and $<3 \mathrm{ng} / \mathrm{mL}$, respectively (19; squares). Neurologic dysfunction was classified according to the MFS as follows: paraspinal hyperesthesia only (grade 5), ambulatory paraparesis and ataxia (grade 4), nonambulatory paraparesis (grade 3), paraplegia with intact nociception (grade 2), paraplegia with no superficial nociception (grade 1), and paraplegia with no deep nociception (grade 0).

In affected dogs, the odds (95\% CI, 1.6 to 757) of longterm voluntary ambulation in those for which CSF CK activity was $\leq 38 \mathrm{U} / \mathrm{L}$ was 35 times the odds in those for which CSF CK activity was $>38 \mathrm{U} / \mathrm{L}(P=0.024)$. The odds (95\% CI, 1.9 to 554) of long-term voluntary ambulation in affected dogs that had a CSF MBP concentration $\leq 3 \mathrm{ng} / \mathrm{mL}$ was 33 times the odds in affected dogs that had a CSF MBP concentration $>3 \mathrm{ng} / \mathrm{mL}$ ( $P$ $=0.016)$. On the basis of the multivariable logistic regression model, the probability of a successful outcome for dogs with an initial MFS of 0 or 1 and CSF CK activity and MBP concentrations values greater than the established cutoffs was $<10 \%$ (Figure 1). Conversely, the probability of a successful outcome was $>98 \%$ for dogs with CSF CK activity and MBP concentration values less than the cutoffs, regardless of initial MFS. The final multivariate model was a good fit to the data on the basis of the Hosmer and Lemeshow test $\left(\chi^{2}=1.12\right.$; $P=0.993$ ).

\section{Discussion}

In the study reported here, CSF CK activity was associated with long-term outcome in dogs with acute thoracolumbar IVDH. Median CSF CK activity was significantly higher in dogs that had an unsuccessful outcome (62 U/L), compared with that in dogs without thoracolumbar IVDH $(<20 \mathrm{U} / \mathrm{L})$ and dogs with thoracolumbar IVDH that had a successful outcome (20 U/L). Additionally, the odds of recovery for dogs with thoracolumbar IVDH that had a CSF CK activity $\leq 38$ U/L was greater (35-fold difference) than the odds for affected dogs that had a CSF CK activity $>38 \mathrm{U} / \mathrm{L}$, adjusting for initial MFS and CFS MBP concentration.

Creatine kinase is a mitochondrial-associated enzyme involved in energy metabolism in the CNS. There are 3 potential causes for increased activity of $\mathrm{CK}$ in the CSF: contamination of CSF by plasma during sample collection, breakdown of the blood-brain barrier with the CK source being plasma, or direct leakage of CK from neuroparenchyma due to cellular dysfunction and damage. ${ }^{25,26}$ Previous evaluations of CK activity in CSF samples obtained from humans and other animals (horses, dogs, and cats) have revealed variable diagnostic and prognostic usefulness. Although findings of 1 study $^{27}$ indicated that CSF CK activity was high in humans who were dying as a result of a stroke, another study ${ }^{17}$ revealed no diagnostic or prognostic value of CSF CK activity for a myriad of CNS diseases in humans. In veterinary medicine, CSF CK activity in dogs with CNS disease was correlated with a high mortality rate $(81 \%)$ when CK activity was $>20$ U/L. ${ }^{28}$ However, all 12 dogs with IVDH in that study ${ }^{28}$ had CK activities $\leq 10 \mathrm{U} / \mathrm{L}$, and long-term outcome in those dogs was not stated. In another report, ${ }^{29}$ high CSF CK activity was associated with poor recovery in animals that had seizures or suspected poisoning. In dogs with IVDH in that study, ${ }^{29}$ no significant association was found between CSF CK activity and outcome; however, only 2 dogs had IVDH. In a recent investigation ${ }^{16}$ involving 97 dogs with thoracolumbar disk disease, CSF CK activity was significantly higher in affected dogs, compared with that in controls; unfortunately, correlation with functional recovery was not reported.

Variability in CSF sample collection and handling may explain inconsistent associations between CSF CK activity and outcome in relation to various CNS diseases. ${ }^{18,27-29}$ In humans, CK activity in serum is highly unstable. Conditions such as freezing; exposure to high ambient temperature, light, or carbon dioxide; oxidation; and serum sample storage duration have been shown to result in artificially decreased CK activity. ${ }^{30}$ Because many researchers have assessed CK activity in archival, frozen CSF samples, an adjunct experiment 
was performed by the authors to determine whether frozen samples could be used as surrogates for fresh CSF samples. In fresh and frozen CSF samples from 10 dogs with IVDH, there was no correlation between values of CK activity. This information, in agreement with previous reports, ${ }^{17,30}$ suggested that CK in CSF samples is unstable; hence, the CSF biomarker analyses in the present study were performed rapidly after CSF sample acquisition without freezing of those samples.

In the study reported here, CSF CK activity was a useful outcome predictor when combined with CSF MBP concentration and the MFS. Both the MFS' ${ }^{23}$ and CSF MBP concentration ${ }^{13}$ have been previously associated with functional recovery in dogs with thoracolumbar IVDH and were shown via the logistic regression analysis to be independently related to outcome within the dogs in the present study; details of the findings regarding MBP concentration have been communicated in another report ${ }^{13}$ about the same group of dogs. The collective impact of CSF CK activity, CSF MBP concentration, and MFS depends on case characteristics. For example, in a dog with an MFS of 0 or 1 for which both CSF MBP concentration and CSF CK activity are greater than the respective cutoff value, the probability of long-term ambulatory recovery was only 6\% (Figure 1). However, in this same MFS category, when CSF MBP concentration and CSF CK activity were both less than the respective cutoff value, functional recovery was $>98 \%$. It appears likely that by use of multiple independent outcome predictors in dogs with thoracolumbar IVDH, prognosis can be more effectively determined than it can on the basis of results of nociceptive assessment alone.

Other CSF biomarkers assessed in the present study included nucleated cell count, AST activity, and total protein, lactate, glucose, and calcium concentrations. Cerebrospinal fluid nucleated cell count was significantly higher in dogs with IVDH, compared with that in control dogs, likely because of inflammatory secondary spinal cord injury. Although the presence of CSF pleocytosis was significantly more frequent in dogs with thoracolumbar IVDH that had unsuccessful outcome, compared with dogs with thoracolumbar IVDH that had functional recovery, CSF pleocytosis was not predictive of outcome when adjusted for initial MFS in our multivariable logistic regression models. Glucose concentration was significantly higher in the CSF of dogs with thoracolumbar IVDH, compared with that of control dogs. This may be related to breakdown of the blood-CSF barrier in dogs with disk-associated spinal cord injury or could be attributable to signalment differences within the control and affected groups. Cerebrospinal fluid total protein, lactate, and calcium concentrations and AST activity were not related to group or to outcome in dogs with thoracolumbar IVDH. Additionally, signalment, preoperative administration of glucocorticoids or NSAIDs, and plasma biomarker concentrations and activities were not related to functional recovery in dogs with thoracolumbar IVDH, similar to results of previous studies. ${ }^{1,11,31}$

Plasma and CSF concentrations of calcium, glucose, and lactate were correlated with each other, but AST and $\mathrm{CK}$ activities were not correlated. The authors believe the most likely explanation is that AST and CK are enzymes that have a much larger molecular size than that of the other assessed biomarkers, which potentially limited their diffusion across the blood-brain barrier. Further research is needed to evaluate this hypothesis.

The potential limitations of the study reported here include the method of in-hospital neurologic evaluation, relatively small group sizes, single-center design, and acquisition of only CSF samples from the cerebromedullary cistern. In-hospital evaluation was limited to neurologic examination performed by use of a validated scoring system; computerized gait analy$\mathrm{sis}^{32}$ and assessment of somatosensory evoked and motor evoked potentials ${ }^{33}$ may have provided additional useful data. Also, surrogate markers of recovery such as fecal and urinary continence were not examined. Importantly, the power of the present study to detect small or moderate effects on outcome may have been limited, especially considering the small number of dogs with unsuccessful outcomes (9/54). A multicenter approach would help confirm the data obtained in the present study, facilitate the adjustment of confounding variables, and increase the numbers of available dogs for evaluation. Differences in signalment between the control and affected groups may have biased biomarker comparisons because breed, sex, or age matching was not possible. Finally, lumbar CSF samples were not obtained in the study. It is possible that biomarker concentrations or activities may differ on the basis of the site of CSF sample acquisition and that outcome associations determined in the present study may not be representative of lumbar CSF sample findings.

In the present study, high CSF CK activity was a negative predictor of long-term ambulatory outcome in dogs with acute thoracolumbar IVDH. However, CSF CK activity, CSF MBP concentration, and MFS appeared to be independent predictors, which could be used collectively to improve the precision of prognosis. These novel data in a single group of dogs can be used as a starting point for future prospective studies investigating the clinical usefulness of CSF CK activity and MBP concentration as prognostic indicators in dogs with acute thoracolumbar IVDH.

\footnotetext{
a. Rapinovet, Schering-Plough Animal Health Corp, Union, NJ.

b. SevoFlo, Abbott Laboratories, North Chicago, Ill. IsoFlo, Abbott Laboratories, North Chicago, Ill.

d. Vitros 250, Ortho-Clinical Diagnostics Inc, Rochester, NY.

e. SPSS, version 17.0, SPSS Inc, Chicago, Ill.

f. Epi Info, version 6.04, CDC, Atlanta, Ga.
}

\section{References}

1. Ferreira AJ, Correia JH, Jaggy A. Thoracolumbar disc disease in 71 paraplegic dogs: influence of rate of onset and duration of clinical signs on treatment results. I Small Anim Pract 2002;43:158-163.

2. Davis GJ, Brown DC. Prognostic indicators for time to ambulation after surgical decompression in nonambulatory dogs with acute thoracolumbar disk extrusions: 112 cases. Vet Surg 2002;31:513-518.

3. Gambardella P. Dorsal decompressive laminectomy for treatment of thoracolumbar disc disease in dogs: a retrospective study of 98 cases. Vet Surg 1980;9:24-26.

4. Sukhiani HR, Parent JM, Atilola MA, et al. Intervertebral disk disease in dogs with signs of back pain alone: 25 cases (19861993). IAm Vet Med Assoc 1996;209:1275-1279. 
5. Scott H. Hemilaminectomy for the treatment of thoracolumbar disc disease in the dog: a follow-up study of 40 cases. I Small Anim Pract 1997;38:488-494.

6. Duval J, Dewey C, Roberts R, et al. Spinal cord swelling as a myelographic indicator of prognosis: a retrospective study in dogs with intervertebral disc disease and loss of deep pain perception. Vet Surg 1996;25:6-12.

7. Laitinen OM, Puerto DA. Surgical decompression in dogs with thoracolumbar intervertebral disc disease and loss of deep pain perception: a retrospective study of 46 cases. Acta Vet Scand 2005;46:79-85.

8. Scott HW, McKee WM. Laminectomy for 34 dogs with thoracolumbar intervertebral disc disease and loss of deep pain perception. I Small Anim Pract 1999;40:417-422.

9. Anderson SM, Lippincot CL, Gill PJ. Hemilaminectomy in dogs without deep pain perception. Calif Vet 1991;45:24-28.

10. Loughin CA, Dewey CW, Ringwood PB, et al. Effect of durotomy on functional outcome of dogs with type I thoracolumbar disc extrusion and absent deep pain sensation. Vet Comp Orthop Traumatol 2005;18:141-146.

11. Ito D, Matsunaga S, Jeffery ND, et al. Prognostic value of magnetic resonance imaging in dogs with paraplegia caused by thoracolumbar intervertebral disk extrusion: 77 cases (20002003). I Am Vet Med Assoc 2005;227:1454-1460.

12. Levine JM, Fosgate GT, Chen AV, et al. Magnetic resonance imaging in dogs with neurologic impairment due to acute thoracic and lumbar intervertebral disk herniation. I Vet Intern Med 2009;23:1220-1226.

13. Levine GJ, Levine JM, Witsberger TH, et al. Cerebrospinal fluid myelin basic protein as a prognostic biomarker in dogs with thoracolumbar intervertebral disk herniation. I Vet Intern Med 2010;24:890-896.

14. Thomson CE, Kornegay JN, Stevens JB. Canine intervertebral disc disease: changes in the cerebrospinal fluid. I Small Anim Pract 1989;30:685-688.

15. Anderson DK, Prockop LD, Means ED, et al. Cerebrospinal fluid lactate and electrolyte levels following experimental spinal cord injury. INeurosurg 1976;44:715-722.

16. Necas A, Sedlakova D. Changes in the creatine kinase and lactate dehydrogenase activities in cerebrospinal fluid of dogs with thoracolumbar disc disease. Acta Vet (Brno) 1999;68:111-120.

17. Lisak RP, Graig FA. Lack of diagnostic value of creatine phosphokinase assay in spinal fluid. IAMA 1967;199:750-751.

18. Jackson C, de Lahunta A, Divers T, et al. The diagnostic utility of cerebrospinal fluid creatine kinase activity in the horse. $L$ Vet Intern Med 1996;10:246-251.

19. Osuna E, Perez-Carceles MD, Luna A, et al. Efficacy of cerebro- spinal fluid biochemistry in the diagnosis of brain insult. Forensic Sci Int 1992;52:193-198.

20. Satoh H, Yamato O, Asano T, et al. Cerebrospinal fluid biomarkers showing neurodegeneration in dogs with GMI gangliosidosis: possible use for assessment of a therapeutic regimen. Brain Res 2007;1133:200-208.

21. Hurtt AE, Smith MO. Effects of iatrogenic blood contamination on results of cerebrospinal fluid analysis in clinically normal dogs and dogs with neurologic disease. I Am Vet Med Assoc 1997;211:866-867.

22. Levine GJ, Levine JM, Budke CM, et al. Description and repeatability of a newly developed spinal cord injury scale for dogs. Prev Vet Med 2009;89:121-127.

23. Levine JM, Ruaux CG, Bergman RL, et al. Matrix metalloproteinase-9 activity in the cerebrospinal fluid and serum of dogs with acute spinal cord trauma from intervertebral disk disease. Am I Vet Res 2006;67:283-287.

24. Windsor RC, Vernau KM, Sturges BK, et al. Lumbar cerebrospinal fluid in dogs with type I intervertebral disc herniation. I Vet Intern Med 2008;22:954-960.

25. Wilson JW, Wiltrout SK. Cerebrospinal fluid creatine phosphokinase in the normal dog. Am I Vet Res 1976;37:1099-1100.

26. Inao S, Marmarou A, Clarke GD, et al. Production and clearance of lactate from brain tissue, cerebrospinal fluid, and serum following experimental brain injury. INeurosurg 1988;69:736-744.

27. Vaagenes P, Urdal P, Melvoll R, et al. Enzyme level changes in the cerebrospinal fluid of patients with acute stroke. Arch Neurol 1986;43:357-362.

28. Indrieri RJ, Holliday TA, Keen CL. Critical evaluation of creatine phosphokinase in cerebrospinal fluid of dogs with neurologic disease. Am I Vet Res 1980;41:1299-1303.

29. Wilson JW. Clinical application of cerebrospinal fluid creatine phosphokinase determination. I Am Vet Med Assoc 1977;171:200-202.

30. Moss D, Henderson A. Enzymes. In: Burtis C, Ashwood E, eds. Tietz textbook of clinical chemistry. 2nd ed. Philadelphia: Saunders, 1994;803-805.

31. Olby N, Levine J, Harris T, et al. Long-term functional outcome of dogs with severe injuries of the thoracolumbar spinal cord: 87 cases (1996-2001). I Am Vet Med Assoc 2003;222:762-769.

32. Hamilton L, Franklin RJ, Jeffery ND. Development of a universal measure of quadrupedal forelimb-hindlimb coordination using digital motion capture and computerised analysis. BMC Neurosci [serial online] 2007;8:77. Available at: www.biomedcentral. com/1471-2202/8/77. Accessed Apr 18, 2010.

33. Poncelet L, Michaux C, Balligand M. Somatosensory potentials in dogs with naturally acquired thoracolumbar spinal cord disease. Am I Vet Res 1993;54:1935-1941. 